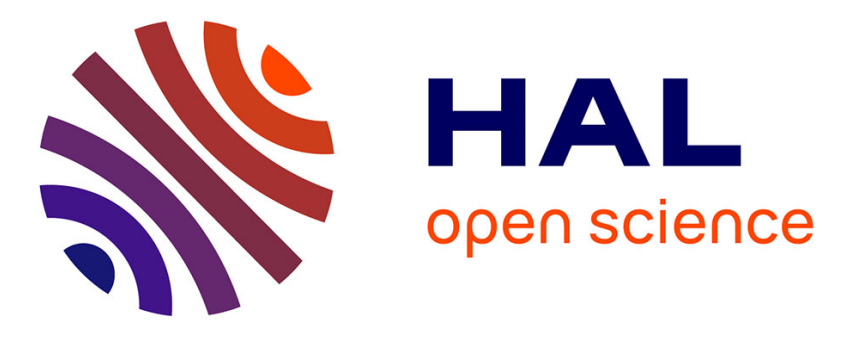

\title{
Improving Collaboration Between Large and Small Enterprises Using Networked Services
}

Wolfgang Gräther, Isabel Matranga, Vincenzo Savarino, Karol Furdik, Martin Tomášek

\section{- To cite this version:}

Wolfgang Gräther, Isabel Matranga, Vincenzo Savarino, Karol Furdik, Martin Tomášek. Improving Collaboration Between Large and Small Enterprises Using Networked Services. 16th Working Conference on Virtual Enterprises (PROVE), Oct 2015, Albi, France. pp.201-208, 10.1007/978-3319-24141-8_18. hal-01437888

\section{HAL Id: hal-01437888 \\ https://hal.inria.fr/hal-01437888}

Submitted on 17 Jan 2017

HAL is a multi-disciplinary open access archive for the deposit and dissemination of scientific research documents, whether they are published or not. The documents may come from teaching and research institutions in France or abroad, or from public or private research centers.
L'archive ouverte pluridisciplinaire HAL, est destinée au dépôt et à la diffusion de documents scientifiques de niveau recherche, publiés ou non, émanant des établissements d'enseignement et de recherche français ou étrangers, des laboratoires publics ou privés.

\section{(c)(1)}

Distributed under a Creative Commons Attribution| 4.0 International License 


\title{
Improving Collaboration between Large and Small Enterprises Using Networked Services
}

\author{
Wolfgang Gräther ${ }^{1}$, Isabel Matranga ${ }^{2}$, Vincenzo Savarino², Karol Furdik ${ }^{3}$, Martin \\ Tomášek ${ }^{3}$ \\ ${ }^{1}$ Fraunhofer FIT, St. Augustin, Germany, wolfgang.graether@fit.fraunhofer.de \\ ${ }^{2}$ Ingegneria Informatica, Palermo, Italy \{isabel.matranga, vincenzo.savarino\}@eng.it \\ ${ }^{3}$ Intersoft, a.s., Kosice, Slovakia \{karol.furdik, martin.tomasek\}@intersoft.sk
}

\begin{abstract}
Collaboration between large and small \& medium-sized enterprises is still not adequately supported by current groupware solutions. In this paper, we present a novel approach that addresses the challenges of inter-enterprise collaboration. Key elements of our approach are: interoperability to legacy applications to retain current working styles, basic services for sharing and management of shared collaboration spaces, use of email for collaboration especially on the SME's side, information extraction to enable semantic search in inter-enterprise collaborations and automatic tagging of documents, and integration of business process models. We evaluated the approach on three application cases in the workplace: new product development, software development supply chain, and supply chain collaboration between SMEs and LEs. Our results suggest that the implemented services are beneficial in typical cooperation situations between enterprises.
\end{abstract}

Keywords: Groupware, interoperability, lightweight semantics, virtual enterprise.

\section{Introduction}

Collaboration between large enterprises (LE) and small and medium-sized enterprises (SME) is often solely based on exchanging documents via email. The advantage of email usage is the interoperability of email clients, which means that it is straightforward and easy to distribute documents and other information to co-workers even if they are using different email clients. There are drawbacks with email usage: for example, email usage could lead to divergent document versions, does not provide awareness about activities of co-workers, and could overwhelm users with messages.

However, even if some of the cooperating enterprises are using collaboration suites (groupware), there exist several reasons to collaborate via email:

- the cooperating enterprises are using different collaboration systems that are not interoperable,

- even if the cooperating partners agree on a common collaboration system, then especially SMEs often do not have the resources to buy, install and use all the different collaboration suites of their LE partners, 
- if one partner is already using a collaboration system, then often external partners are not allowed to register with the partner's collaboration suite,

- the compliance rules of the cooperating enterprises do not allow to use services in the cloud, such as Doodle for scheduling or Dropbox for sharing.

In this paper, we present a novel solution to this interoperability problem. After a section with related work, we will report the details of our approach. The description of the VENIS application cases, the method and settings for the evaluation as well as the presentation of the evaluation results follow. Finally, we present a conclusion and future work.

\section{Related Work}

Interoperability of collaborative working environments (CWE) has been previously studied and prototypically tested in several research projects. A Reference architecture for interoperable CWE systems as well as concrete implementations can be found in [1] and [2]. This generic architecture is still inspiring; however, our approach focuses on integration of modularized services and connects to enterprise repositories and legacy systems.

Email is still widely used today even if there are problems and implications of message-based patterns for organizing collaboration [3, 4]. For example, exchange of documents as email attachments cause extra coordination work for the co-workers and multiple copies of the documents are stored in the users' email inboxes. In addition, information overload could be a problem especially when distribution lists are used. This research supports our decision to integrate email in inter-enterprise collaborations.

In [5] a model for automatic suggestion of shared spaces when a user composes an email is presented, i.e. email is posted to relevant shared spaces such as wikis, for example. This approach preserves email usage as our approach does, but we go further and integrate attachment stripping and named entity recognition to support semantic search. Our work on email analysis, information extraction, enterprise search, and lightweight semantics has been reported earlier [6-9].

\section{The VENIS Approach}

The requirements for the VENIS approach were elicited from an analysis of LE-SME collaboration settings and the three application cases. These requirements informed our design that was mainly driven by the desire to combine the ad hoc features of email, lightweight semantic technologies, and business process support into distributed services to enabling interoperability and collaboration between enterprises.

The VENIS services for interoperability (VSI) are included in so-called VSI nodes, which are connected in a network, see Fig. 1. Usually each collaborating enterprise hosts its own VSI node, but, for example, very small SMEs could be invited to connect to a VSI node of an enterprise they are collaborating with. At the network 
level, the VENIS network administrator manages the Yellow Pages Server and keeps the network configuration up to date.

The VENIS services for interoperability comprise basic groupware services: sharing of artifacts, management of shared collaboration spaces, membership management, and means to notify members about ongoing activities of co-workers. The traditional login-password access mechanism is replaced by tokens, which enable collaboration members to access and to revise shared artifacts as well as to upload new artifacts.

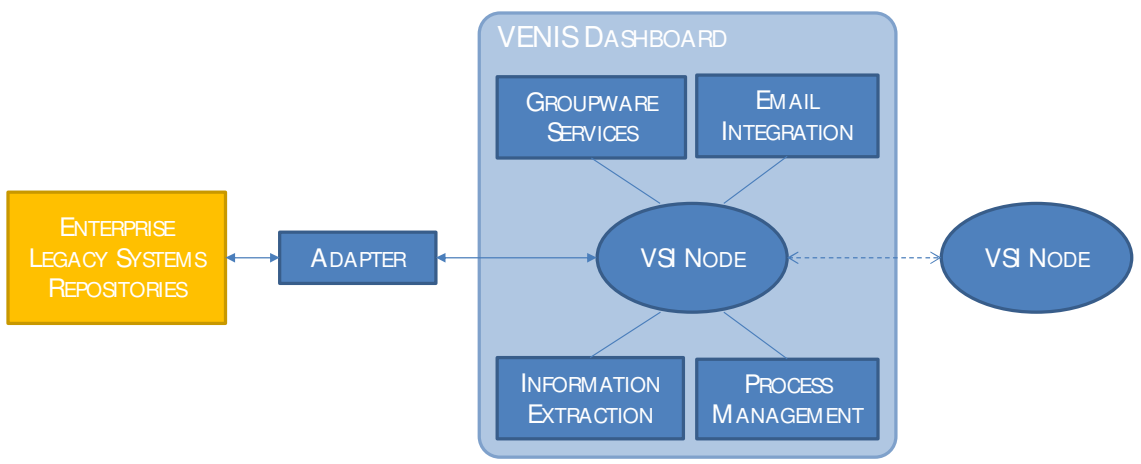

Fig. 1. Conceptual model and components of VENIS.

The VENIS approach integrates email into collaborative processes. For example, tokens to access artifacts are sent via email. In addition, attached documents are automatically stripped of and become thus part of the addressed collaboration space. Furthermore, email conversations are input for indexing and information extraction.

The means for searching are based on indexing and information extraction services from emails and human-readable documents in collaborative processes. The extracted semantic information is stored in a semantic network as a free collection of types. In earlier research work [9] default annotation types for business documents have been identified: organization, person, address, product, document, inventory, etc. The type organization, for example, is sub-structured into attributes such as name, organization identifier and tax registration number. The type of a document could be invoice, order, contract, or change request. The extracted semantic information enables rich semantic search over emails and collaboration artifacts.

The VENIS approach facilitates usage of business process models in collaborative processes, i.e. management and control of the collaboration flow is supported by notifications and task lists. Assume, for example, that a LE-SME business process model has been created, then the execution triggers an event and an email will be sent to the SME employee containing the token for a particular document. The SME will receive the token and will get access to the business document to work on it. After finishing the work and uploading the updated document, other co-workers are informed and the business process continues with the next steps. 
There is, of course, a graphical user interface - the VENIS dashboard - available that offers all basic groupware functions, search functions as well as functions for task management to co-workers and it displays an activity stream.

The concept of adapters is used to connect VENIS services to enterprise repositories and legacy systems. Currently there is a basic set of adapters available that seems to be sufficient for SMEs: FTP, WebDav, SQL data bases, etc. The Engineering's application case 'new product development' uses an adapter to connect to the portal and collaboration software Liferay. From an end-user's perspective, adapters help preserve current working styles.

For a detailed description of the VENIS approach, please refer to the project documents 'D5.3 VSI Online' and 'D6.2 VSI Prototype' [10, 11] and videos [11].

\section{Style of Working and Description of Application Cases}

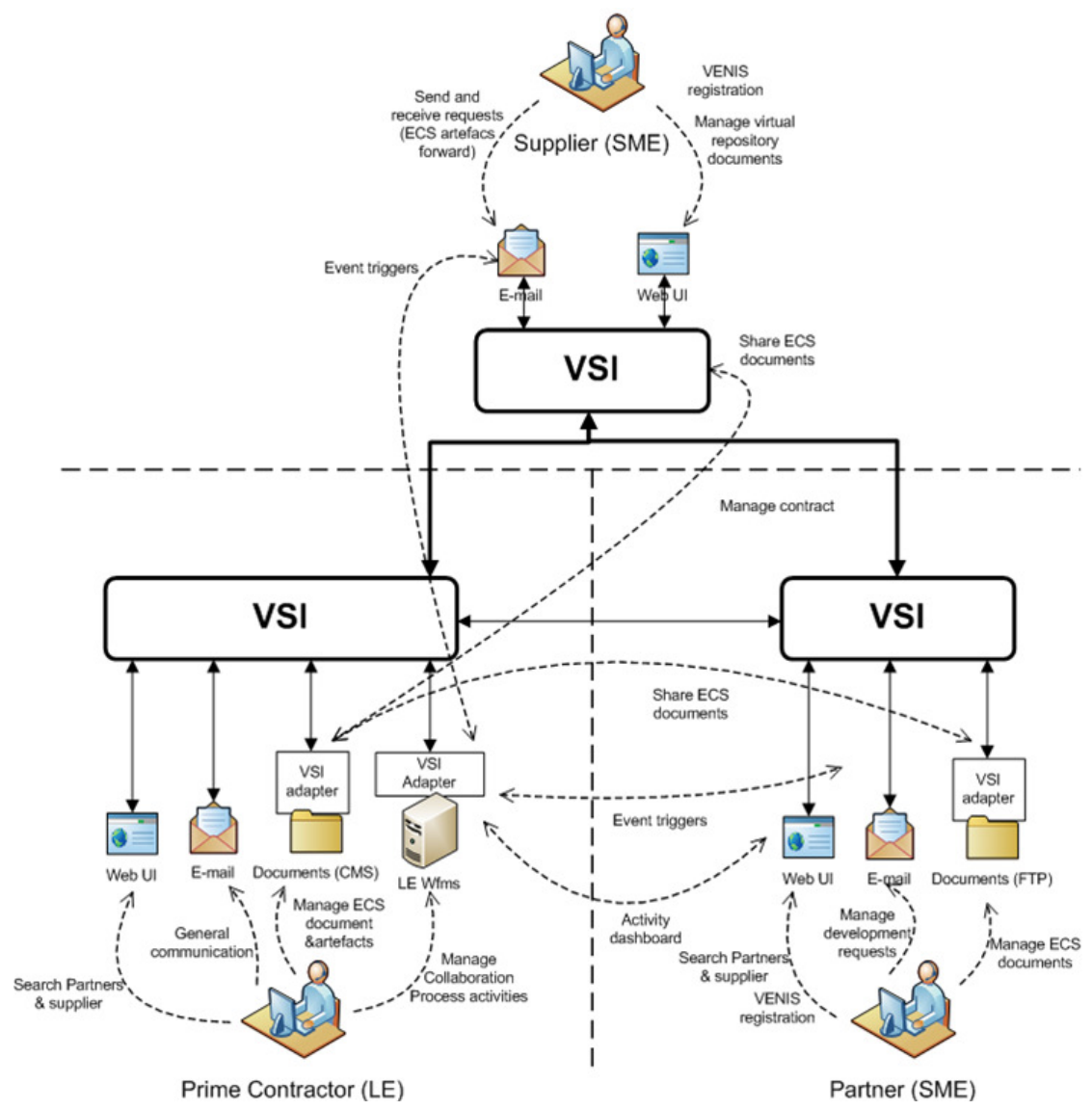

Fig. 2. Application case 'New product development'. 
The style of working for LEs and SMEs differ because large enterprises usually handle business processes and documents in a more formalized way. An ideal case of interoperability would be the exchange of business data via standardized documents, for example, using CoreComponents, EDI or ebXML. However, SMEs often handle business documents in human-readable format such as doc, pdf or html and the fixed working procedures driven by legacy applications are missed. These procedures are replaced by the SME employee's decisions and actions taken according to the usual unwritten way of working [6].

The three application cases reflect these different working practices. For example, the application case depicted in Fig. 2 focuses on the exchange of resources between SMEs and LE and the interaction of distributed working groups within a business process. Collaboration steps between a prime contractor, a partner company and a supplier are shown.

The application case 'software development supply chain' illustrates the situation that a provider, requiring an implementation of a complex software project, needs additional resources from suppliers. In this application case the VENIS support focuses on the quick identification of suppliers by their skills and on easy management of technical and contractual aspects between provider and supplier.

The application case 'supply chain collaboration between SMEs and LE' stands for typical inter-enterprise collaboration. The SME supplies IT and Telematics software and hardware to the large enterprise as well as staff when required so as to fulfill the needs of their control room staffing requirements. The collaboration includes procedures of getting quotes and orders from the LE to the SME, while the SME provides either quotes or fulfills the orders that have been sent.

Altogether, the three application cases are typical for current LE and SME collaboration and require almost all features that are available in the VENIS services for enterprise interoperability: adapters to connect to legacy systems and enterprise repositories, basic groupware services, email for messaging and token-based access to shared documents, functions for searching and business process support.

\section{Evaluation Method and Settings for Application Cases}

The VENIS approach was evaluated for all three application cases. The goal was to get findings about usability, user friendliness, benefits, and finally an overall business applicability of the VENIS approach as a whole. To evaluate these aspects, an on-line questionnaire was used. The questionnaire was designed with the aim to cover all phases of the software installation, configuration, deployment, and daily operation from various user perspectives. To evaluate the usability in all the identified phases, three user roles and their responsibilities were distinguished as follows:

- Technical system administrator - is responsible for the overall installation, configuration, and deployment of the VENIS network, as well as for the operational technical maintenance.

- Collaboration owner and administrator - uses the installed VENIS dashboard to perform actions needed for set up and maintenance of 
collaborations, including management of collaboration members, handling of business process models and the repository of artifacts.

- Involved collaboration member - participates in an established VENIS collaboration. By means of token links, this user role has a controlled $\mathrm{read} / \mathrm{write}$ access to the shared space of artifacts, is notified about the required tasks specified in business process models or by owner's commands.

In this paper we restrict the presentation of results to the user roles collaboration owner and collaboration member, therefore we describe only these parts of the online questionnaire. The questions for collaboration owners is composed by a block of 13 basic quantitative data (number of created collaborations, number of invited users, number of tasks specified in business process models, etc.) and 16 questions referring to collaboration set up, VENIS dashboard, actions for collaboration management, and perceived security, usefulness and overall satisfaction.

The questions for collaboration members is composed by a block of 13 basic quantitative data (number of accepted invitations to collaborations, duration of involvement in collaborations, number of email notifications, number of artifacts received via token links, etc.) and 23 questions referring to involvement into collaborations, work within collaborations, navigation and VENIS dashboard, and also perceived security, usefulness and overall satisfaction.

For each use case one application partner was responsible for the evaluation and had to set up and customize the corresponding VENIS network. For example, the setting for the application case 'software development supply chain' comprised two networked VSI nodes. Two SME partners and one LE partner have accessed a shared space of collaborations, employing adapters to connect to respective local resource repositories such as TestTrack application, local file system, and FTP space. The selection of partners was performed taking also into account their experience with tools and platforms used for collaboration, so as to enable them to make an effective comparison and evaluation of the VENIS services vs. other tools and platforms used.

\section{Results from Evaluation}

During winter 2015 the three VENIS networks were used by 24 people; one application case involved 12 people, the other two involved 6 users each. 14 shared spaces for collaborations were created and 120 artifacts were stored. Two application cases used the support for business process models with 4 tasks on average.

The evaluation confirmed that the VENIS services could be successfully used for all the three application cases and that usage of the VSI network has been beneficial (overall effective and useful) to the different collaborative business processes. However, the ratings from involved small and medium-sized enterprises were better than that from the large enterprise, which is only little better than neither agree nor disagree. Moreover, the evaluation results indicate that the VENIS services are generally applicable to a large amount of typical collaboration situations involving large and small and medium-sized enterprises through its adaptability, integration of email and business process models. For example, results from application case 
'supply chain collaboration between SMEs and LE' indicate that request for purchasing processes seem to be adequately supported by automating manual tasks.

The deep integration of email into collaborative processes is beneficial for all enterprises. Sharing of artefacts by sending them as attachments was mentioned by the large enterprise as most frequently used method, the SMEs rely on token-based upload of documents.

Tokens are used to access or upload documents, with the advantage that no login is needed. The tokens are sent via email that implies a certain security risk. Despite this situation our evaluation shows a rather positive rating and also confirms the usefulness of the tokens for uploading documents.

The means for searching are based on indexing and information extraction services and are automatically performed on email and other human-readable documents that are shared in collaborative processes. Those services support annotation of documents and enable full text as well as semantic search. The evaluation shows for collaboration owners as well as for collaboration members a slight superiority of full text search over the innovative semantic search.

The evaluation of the means for business process modeling and management of the collaboration flow shows that the users see the potential of the business process support. The robustness and easy to use aspect of the business process support is slightly positive. For the participating SMEs the evaluation indicates that the VENIS services are able to manage contractual aspects, support purchasing processes and make interaction with suppliers easy than what it is in the usual way of working. For the participating large enterprise the results for those questions are neither positive nor negative.

\section{Conclusion and Future Work}

Conducting the evaluation with three application cases provided valuable insights from our users and suggestions how to further improve the VENIS services for interoperability. For example, the overall evaluation of the VENIS dashboard was positive but additional functions such as move, copy, or mark as read for artifacts were requested. The integrated support of business process models was appreciated, but the results suggest simplifying the creation of the process models. The semantic search seems to be too complex for collaboration members and an improved user interaction is requested.

Currently the project team is working on improving the VENIS services. We believe that the VENIS approach combining email, lightweight semantics and business process support in a unified manner has sufficient potential to improve current styles of working between large and small and medium-sized enterprises. 


\section{Acknowledgement}

Our thanks are due to all partners of the VENIS (Virtual Enterprises by Networked Interoperability Services) project that is partially funded by the EU under grant number 284984. Special thanks go to Marco Alessi, Bruno Casali, Stefan Dlugolinský, Martin Šeleng, Harry Trigazis, and Gicomo Vecere as well as to all test persons for their contribution to this work.

\section{References}

1. Peristeras, V., Fradinho, M., Lee, D., Prinz, W., Ruland, R., Iqbal, K., Decker, S.: CERA: A collaborative environment reference architecture for interoperable CWE systems. Service Oriented Computing and Applications 3(1), 3-23 (2009)

2. Prinz, W., Löh, H., Pallot, M., Schaffers, H., Skarmeta, A., Decker, S.: ECOSPACE Towards an Integrated Collaboration Space for eProfessionals. In 2006 International Conference on Collaborative Computing: Networking, Applications and Worksharing. IEEE press (2006)

3. Prinz, W., Jeners, N., Ruland, R., Villa, M.: Supporting the change of cooperation patterns by integrated collaboration tools. In: Leveraging Knowledge for Innovation in Collaborative Networks. IFIP Advances in Information and Communication Technology, 307, pp. 651--658. Springer, Berlin, Heidelberg (2009).

4. Zhang, A.X., Ackerman, M.S., Karger, D.R.: Mailing Lists: Why Are They Still Here, What's Wrong With Them, and How Can We Fix Them? In: $33^{\text {rd }}$ Annual ACM Conference on Human Factors in Computing Systems, pp. 4009--4018. ACM (2015)

5. Mahmud, L., Matthews, T., Whittaker, S., Moran, T. P., Lau, T.: 2011. Topika: Integrating Collaborative Sharing with Email. In: SIGCHI Conference on Human Factors in Computing Systems; pp. 3161--3164. ACM (2011)

6. Seleng, M., Laclavik, M., Dlugolinsky, S., Ciglan, M., Tomasek, M., Hluchy, L.: Approach for Enterprise Search and Interoperability using Lightweight Semantic. In: Intelligent Engineering Systems (INES), 2014 18th International Conference on, pp. 73--78. IEEE (2014)

7. Dlugolinsky, S., Krammer, P., Ciglan, M., Laclavik, M.: MSM2013 IE Challenge: Annotowatch. Making Sense of Microposts (\# MSM2013), (2013)

8. Laclavik, M., Ciglan, M., Dlugolinsky, S., Seleng, Hluchy, L.: Emails as Graph: Relation Discovery in Email Archive. In: $21^{\text {st }}$ international conference companion on World Wide Web, pp. 84--846. ACM (2012)

9. Laclavik, M., Dlugolinsky, S., Seleng, M., Kvassay, M., Gatial, E., Balogh, Z., Hluchy, L.: Email Analysis and Information Extraction for Enterprise Benefit. Computing and informatics, 30(1), pp. 5--87 (2012)

10. VENIS Deliverables, http://www.venis-project.eu/deliverables

11. VENIS Video, http://www.venis-project.eu/video 